\title{
Analysis of human skin tissue by a skin-meter: A preliminary study
}

\author{
Sergio Mazzulla ${ }^{1}$, Annarita Carino ${ }^{1}$, Antonino Miano², Fiorenzo Mignini ${ }^{2}$, Giovanni Bartolomei ${ }^{3}$, \\ Guglielmo Martino ${ }^{1}$ \\ ${ }^{1}$ Department of Cellular Biology, University of Calabria, Cosenza, Italy \\ ${ }^{2}$ Department of Public Healthy and Experimental Medicine, University of Camerino, Camerino, Italy \\ ${ }^{3}$ Department of Chemistry, University of Calabria, Cosenza, Italy \\ Email: mazzulla@unical.it
}

Received 11 September 2012; revised 12 October 2012; accepted 22 October 2012

\begin{abstract}
The aim of this study is to evaluate the skin elasticity in individuals who practise spinning. Mechanical evaluations were conducted, under environmental and physical established conditions, during a physical training period. A total of 12 volunteers aged $35 \pm 5$ years were enrolled in this study, $(60 \mathrm{~min})$ before (control) and (60 min) after practicing spinning. Skin elasticity and area were measured by a Skin Meter and analysed with the Skin Meter software 1.3. In subjects who practiced spinning, the data shown a significant effect: on elasticity, evaluated as the ratio of final retraction (UA) and final distension (UF), on viscoelasticity express as the ratio delayed distension (UV) and immediate distension (UE); and on measurements of skin area vs control subjects. Skin Meter can be useful for a non-invasive screening of skin condition with also a potential extension in subjects with skin pathologies or to monitor skin oxidative stress in sportive subjects.
\end{abstract}

Keywords: Skin Meter; Elasticity; Skin Parameters; Spinning

\section{INTRODUCTION}

Recently, a great deal of in vivo studies using a bioengineering view point for a mechanical evaluation of epidermal tissue have been performed [1-3]. Biological and physiological aspects of this study is founded on the evaluation of the dermis portion of the skin in sportive subjects, before and after exercising, using the elastomeric method. The instrument used for assessing dermal deformation is based on the measurements of skin parameter [4]. Progressive resistance to deformation, flexibility and elasticity must be adequately balanced to fulfil the ideal tensile properties of the skin. Furthermore, the parameters described are the functional expression of the molecular rearrangements of the skin components, whose structural organisation can be ascribed to noxious cellular phenomena such as the oxidative stress and formation of peroxidation products. The elasticity of skin can be consequently compromised and the dermis portion can present biological and dynamic alterations of the structures. Often the stress involves the structural components at several cutaneous and subcutaneous levels to sum up. Aerobic exercise can increase in oxygen demand by working skeletal muscles causing electron leakage from the mitochondria during the movement [5-7]. The aims in the present work, is to test whether the skin property is affected by physical exercise (spinning) and underling possible alterations in physiological mechanisms at skin levels.

\section{MATERIAL AND METHODS}

\subsection{Subject}

Twelve healthy and physically active volunteers: 7 males, $40 \pm 5$ years old, body weight $70 \pm 5 \mathrm{~kg}$ and height $170 \pm$ $8 \mathrm{~cm}$, and 5 females, $40 \pm 5$ years old, weight $60 \pm 5 \mathrm{~kg}$ and height $160 \pm 7 \mathrm{~cm}$. Mean BMI was $25.2 \mathrm{~kg} / \mathrm{m}^{2}$ for male and $21.3 \mathrm{~kg} / \mathrm{m}^{2}$ for female. Trained subjects, not engaged in competitive sports, were enrolled and studied. The study was approved by the university's ethics committee, all participants provided written informed consent upon entry into the study. The changes in skin mechanical parameters 60 min after training, expressed as either increase or decrease of skin elevation during instrumental measurements, are described in subjects practicing spinning $60 \mathrm{~min}$ before and $60 \mathrm{~min}$ after physical exercise. The volunteers performed the spinning session listening to a compilation of 11 songs lasting $50.5 \mathrm{~min}$ Each track corresponded to a specific phase of the session, the phases being labelled as warm-up, sitting, seated climbing, jumping and winning. In addition to the 
music protocol, the participants were assessed to maintain a pedal stroke cadence that had previously been established for each track, thus the training was performed in one hour. They were admitted to the study after personal and familiar anamnesis and medical visit. The exclusion criteria: acute or chronicle infections, allergy, hypersensitivity or allergy to not steroidal anti-inflammatory drugs (FANS) or medicines. They didn't use for 15 days any topical agent on the test areas and their arms resulted free of pathological findings. Spinning activity monitor was conducted three times a week.

\subsection{Dietary Profile}

All participants received explanation and written instruction for them diet. Subjects were asked to be as accurate as possible in recording the amount and type of food and fluid consumed. A list of common household measures, such as cups and tablespoons and specific information about the quantity of measurements (grams, etc.) was given to each participant. Any questions, ambiguities or omission, regarding the type and amount of food and beverages consumed, were resolved individually and controlled via direct interviews. The diet followed by all subjects was based on a Mediterranean-style [8], these data have been acquired through a specific questionnaire to learn about the eating habits of the same subjects.

\subsection{Skin Meter}

In this study was used a Skin-meter as an instrument to determine the physiological parameters, elasticity or viscoelasticity or distensibility of human skin. Skin meter has been used with the purpose of providing specific information on biological skin parameters and human skin area measurements to obtain information on the condition of a specific skin portion taken into examination. Specifically its measurement is based on the physiological deformation of the skin by method of suction. The assessment is made through the analysis of an electrometric parameter, induced by the displacement of a direct component of the probe circuitry affixed to circular symmetric area of skin under examination. The advantage of this determination is that, unlike the optical method, the color or distribution of the skin microcirculation does not interfere with the performed measurements. The probe of the electromechanical Skin-meter produces a continuous transduced signal to accurately and easily evaluate by a reliable instrument and, it makes a determination that allows us to appreciate directly the memory of the elastic deformation in the area of skin, 2 $\mathrm{mm}$ in diameter, affected by sampling and bounded by the geometry of the probe (Figure 1). This technique allows an optimization of the parameters of mechanical functional state of the skin. This is in accordance with the demands and expectations of improved diagnosis of the main parameters affecting the elasticity of the skin to maintain an appropriate biomechanical response in terms of flexibility and resistance to deformation (visco-elasticity). Suction and data acquisition do not use neither optical nor acoustical method, Skin-meter is able to provide symmetrical elasticity measurements in aging process also regardless of elastic fibers orientation.

\subsection{Evaluation of Skin Parameters}

The mechanical properties of the dermis were determined using a non-invasive, in vivo suction skin elasticity meter equipped with $2-\mathrm{mm}$ measuring probe. The time/strain mode was used with a $5 \mathrm{sec}$. application of a constant negative pressure of $400 \mathrm{mbar}$, followed by a 5 $\mathrm{s}$ relaxation period. Elasticity measurements are made in 10 consecutively repeated cycles spaced by $10 \mathrm{~s}$ between them and analysed before $60 \mathrm{~min}$ and after $60 \mathrm{~min}$ exercise. The instrument is placed during the trial so that the probe is always perpendicular to the forearm on the same subject area. Measurements are made on the ventral forearm area of the subject's attesting in a standard posture of testing for all subjects. A typical deformation curve is illustrated in (Figure 2). The following parameters were analysed: immediate distension (Ue), delayed distension (Uv), final distension (Uf), immediate retraction (Ur), final retraction (Ua). The ratio $\mathrm{Ua} / \mathrm{Uf}$ represents the biological elasticity, while the ratio Uv/Ue represents the biological viscoelasticity. The average values of two measurements were used in subsequent calculations. The curves of the obtained skin deformation were analysed using Skin meter 1.3 software programmed in Cell Physiology Laboratory, Department of Cellular Biology, University of Calabria.

\subsection{Analysis of Skin Area Measurement}

The curves of the obtained skin deformation by spinning athletes are wrapped in graphics area. Measurements were made on the volar forearm, this area was considered to use because the surface is better suitable to the

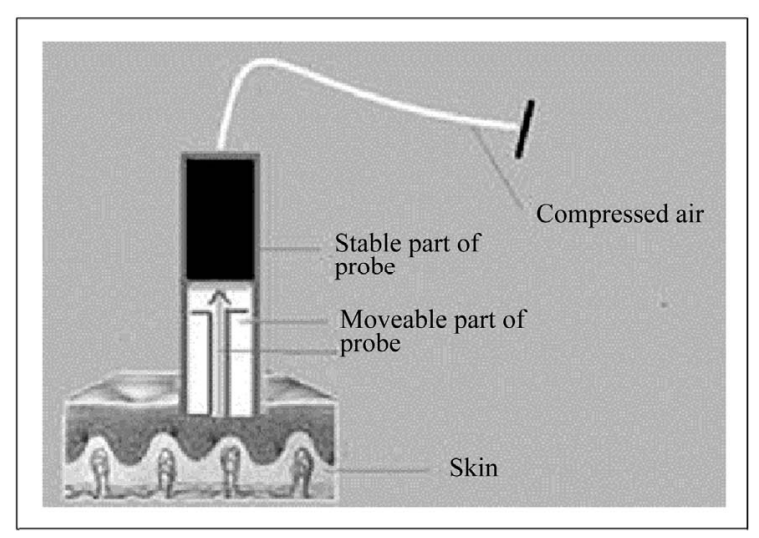

Figure 1. Representation of elastomer probe on human skin. 


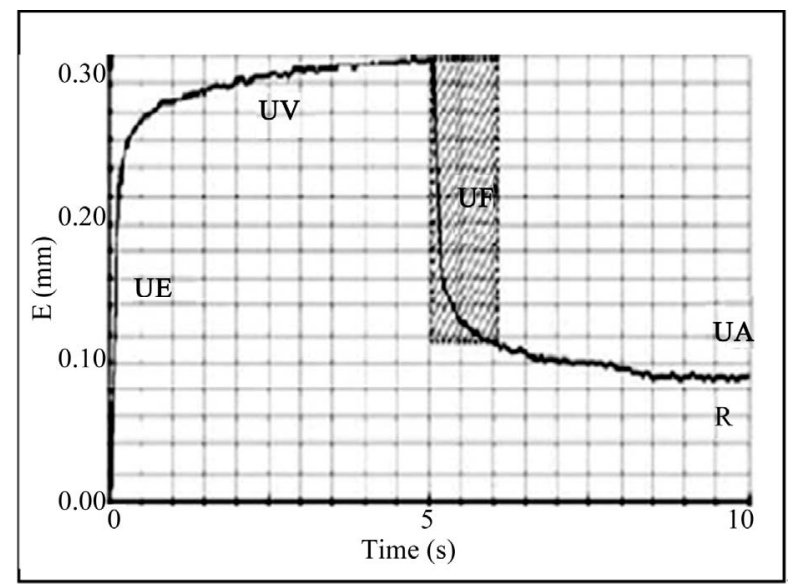

Figure 2. Skin deformation curve.

Skin meter since dermis portion being hairless and slightly vascularized. Area parameters represent another mechanical factor that is affected by physical exercise, and indirectly on the evaluation of skin hydration [9]. These parameters reported as elongation measurements expressed as $(\mathrm{mm})$ calculated for curves taken of the apparatus with a minimum of 10 repetitions in a total time of $100 \mathrm{~s}$ on the specific part of the skin.

\subsection{Statistical Analysis}

The quantitative variables were expressed as mean \pm S.E.M. For each of the parameters, Student's $t$-test for independent observations was used in order to evaluate statistical differences among the two groups with a $p$ under 0.05 as an expression of significance.

\section{RESULTS}

\subsection{Skin Elasticity Measurements}

In subjects who practice spinning we observed a skin deformations suggested by chances of Skin elasticity and viscoelasticity. Significant values of Ua/Uf (elasticity) and $\mathrm{Uv} / \mathrm{Ue}$ (viscoelasticity), measured by $2-\mathrm{mm}$ probe, were significantly increased $60 \mathrm{~min}$ after spinning activity as shown in (Figure 3), in all other skin mechanical parameters the authors did not observe significant changes. In this research, these two skin parameters tested with Skin-meter, show that in athletes, particularly spinning athletes, after training dermis portion manifested an alterability in biological elasticity and in viscoelasticity parameters. Biological elasticity refers to general ratio between elastic and collagen fibres of the tested area, but viscoelasticity is specific for fluid and fibrous biological components of skin, in this research we also observe that the viscoelastic modulus increases, as several studies report [10-12], a change that suggest a indirectly phenomenon of skin dehydration; deformability of the skin results unaltered as deformation after sustained effort. The results of our study indicate that there is no significant difference between males and females in the skin parameter measured before and after exercise.

\subsection{Skin Measurements of Area}

We evaluated steep mode of skin tensile properties in spinning athletes. The repeated cycles of suction-relaxation induced a progressive increase in maximum distension and in residual deformation after spinning activity in many subjects.

The average data for single area tested refer to $0.4 \pm$ $0.16 \mathrm{~mm}$ before spinning activity (Figure 4) and to $0.7 \pm$ $0.13 \mathrm{~mm}$ after spinning activity (Figure 5) in a total time of $100 \mathrm{~s}$.

We noticed that a positive correlation exists because the measurements, developed by Skin-meter, are reproduceible and constant as shown in (Figures 4 and 5).

\section{DISCUSSION}

In the present study the authors used an experimental

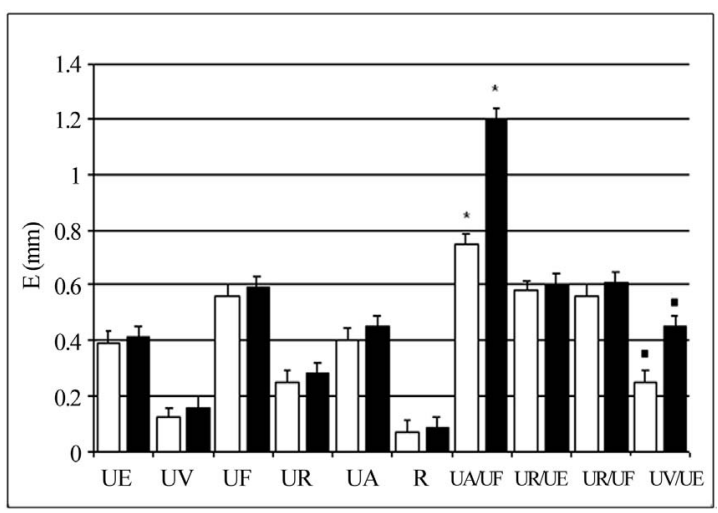

Figure 3. Skin mechanical parameters. \pm S.E.M of ten independent determinations: ${ }^{*} \mathrm{p}<0.05$ (before-after UA/UF), $\mathrm{p}<0.05$ (before-after UV/UE parameters). $\square$ before exercise $\square$ after exercise

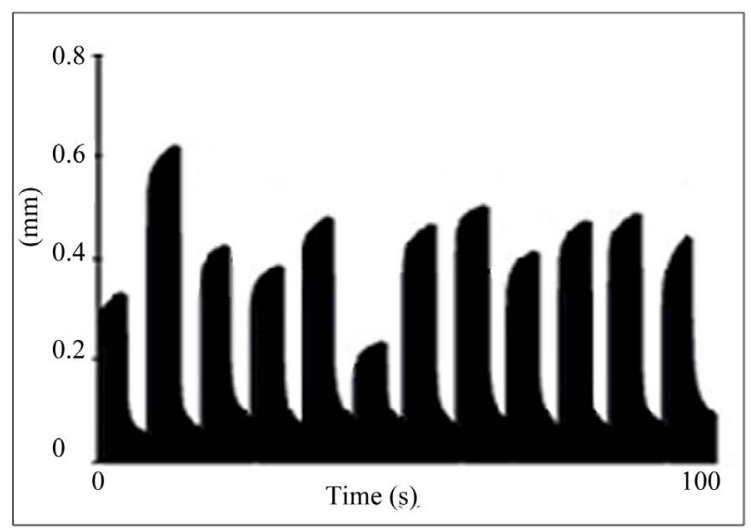

Figure 4. Skin area parameters. Each column is the mean \pm S.E.M. of ten independent determinations (before spinning). 


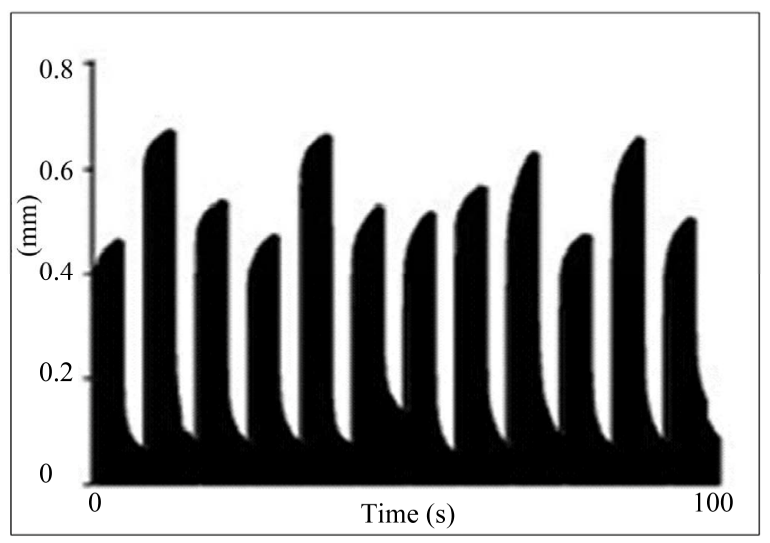

Figure 5. Skin area parameters. Each column is the mean \pm S.E.M. of ten independent determinations (after spinning).

approach to evaluate skin elasticity parameters referring to the practiced sport. Spinning is an aerobic activity founded on a training program, conducted by bicycle movements and continued postural changes. The ventral forearm areas are chosen as that surface are better suited to this type of analysis and easily accessible. That surface is hairless and specific for the task of spinning movement. In the exercise the effort is not made only by the leg muscles, but also by the arm ones, taking in account the different position in the spinning exercise. We have considered it because in the main positions (seated flat, seated climbing, standing flat, standing climbing and jumping) forearm work is present. This area was chosen because best suited to the elastomeric analysis, as the same area is the smoother and more easily accessible to dermal portion useful for the instrument. The change of the electromagnetic field which translates into a digital signal useful to investigate the physical and elastic properties of the skin in spinning subjects. The analysis is founded on the measurements of the electromechanical transduction and the peak area evaluation system by Skin Meter 1.3 software. A key finding of this work is the biological skin response to the physical exercise evaluable by the Skin-meter. In obtained data an increase of elasticity, visco-elasticity and area measurements of the skin are evident after $60 \mathrm{~min}$ of training. The skin rigidity is accompanied by changes not only of the elastic fibers but also of the collagen fibers and the ground substances resulting in an increase of these parameters [13]. In this study, spinning subjects, after exercise, show an improvement in the elastic component, but at the same time, there is a need of a greater moisturing to the skin subjected to exercise.

\section{CONCLUSION}

This preliminary study demonstrates the functionality of Skin-meter and confirms that the measurements not are affected by the optical components of the circuitry with use of a specific probe, so the skin and area measurements are not invalidated by the presence of blood microcirculation of the subjects tested. Skin parameters are also easily detected and analysed, the data prove to be in agreement with results reported in previous studies [12,14-16]. The method used may be effective in a specific way: only dermis portion of human skin is considered and this allows detecting the physiological requirements necessary to the human skin to not be compromised after training. We hypothesize that an important practical implication is that the equipment shall be useful to get a direct monitoring of dermal skin condition in relation to different kinds of sports and to various skin diseases.

\section{REFERENCES}

[1] Urso, M.L. and Clarkson, P.M. (2003) Oxidative stress, exercise, and antioxidant supplementation. Toxicology, 189, 41-54.

http://www.sciencedirect.com/science/article/pii/S030048 3X03001513 doi:10.1016/S0300-483X(03)00151-3

[2] Hwang, E.S. and Kim, G.H. (2007) Biomarkers for oxidative stress status of DNA, lipids, and proteins in vitro and in vivo cancer research. Toxicology, 229, 1-10. doi:10.1016/j.tox.2006.10.013

[3] Lumpkin, E.A. and Caterina, M.J. (2007) Mechanism of sensory transduction in the skin. Nature, 445, 858-865. doi: $10.1038 /$ nature 05662

[4] Dobrev, H. (2000) Use of cutometer to assess epidermal hydratation. Skin Research and Tecnology, 6, 239-244. doi:10.1034/j.1600-0846.2000.006004239.x

[5] Alessio, H.M., Hagerman, A.E., Fulkerson, B.K., Ambrose, J., Rice, R.E. and Wiley, R.L. (2000) Generation of ROS after exhaustive aerobic and isometric exercise. Medicine and Science in Sports and Exercise, 32, 1576-1581. doi:10.1097/00005768-200009000-00008

[6] Bloomer, R.J., Galdfarb, A.H., Wideman, L., McKenzie, M.J. and Consitt, L.A. (2005) Effect of acute aerobic and anaerobic exercise on blood makers of oxidative stress. Journal of Strength and Conditioning Research, 19, 276285.

[7] Fisher-Wellman, K. and Bloomer, R.J. (2009). Acute exercise and oxidative stress: A-30-year review. Dynamic Medicine, 8, 1. doi:10.1186/1476-5918-8-1

[8] Garaulet, M. and Pérez de Heredia, F. (2010) Behavioural therapy in the treatment of obesity (II): Role of the Mediterranean diet. Nutrición Hospitalaria, 25, 9-17. http://scielo.isciii.es/scielo.php?script $=$ sci arttext\&pid $=\mathrm{S}$ 021216112010000100002\&lng=en\&nrm=iso\&tlng=en

[9] Dobrev, H. (2005) Application of Cutometer area parameters for the study of human skin fatigue. Skin Research and Technology, 11, 120-122. doi:10.1111/j.1600-0846.2005.00090.x

[10] Gautieri, A., Pate, M.I., Vesentini, S., Redarelli, A. and 
Buehler, M.J. (2012) Hydratation and distance dependence of intermolecular shearing between collagen molecules in a model microfibril. Journal of Biomechanics, 45. doi:10.1016/i.jbiomech.2012.05.047

[11] Flynn, C., Taberner, A. and Nielsen, P. (2011) Modeling: The mechanical response of in vivo human skin under a rich set of deformation. Annals of Biomedical Engineering, 39, 1935-1946. doi:10.1007/s10439-011-0292-7

[12] Dobrev, H. (2007) In vivo study of skin mechanical properties in Raynoud's phenomen. Skin Research and Technologies, 13, 191-194.

[13] Hermanns-Lê, T., Uhoda, I., Smitz, S., et al. (2004) Skin tensile properties revisited during ageing. Where now, where next. Journal of Cosmetic Dermatology, 3, 35-40. doi:10.1111/j.1473-2130.2004.00057.x
[14] Dobrev, H. (1999) In vivo study of skin mechanical properties in patients with systemic sclerosis. Journal of the American Academy of Dermatology, 40, 436-442.

http://www.sciencedirect.com/science/article/pii/S019096 2299704949

doi:10.1016/S0190-9622(99)70494-9

[15] Dobrev, H. (2002) A study of human skin mechanical properties by means of Cutometer. Folia Medica, 44, 510.

[16] Ono, I. (2011) A Study on the Alterations in Skin Viscoelasticity before and after an intradermal administration of growth factor. Journal of Cutaneous and Aesthetic Surgery, 4, 98-104.

http://dx.crossref.org/10.4103\%2F0974-2077.85022 doi:10.4103/0974-2077.85022 